\title{
BMJ Open Pre-post implementation survey of a multicomponent intervention to improve informed consent for caesarean section in Southern Malawi
}

\author{
Siem Zethof, ${ }^{1,2}$ Wouter Bakker, ${ }^{2,3}$ Felix Nansongole, ${ }^{2}$ Kelvin Kilowe, ${ }^{4}$ \\ Jos van Roosmalen, ${ }^{1,3}$ Thomas van den Akker ${ }^{1,3}$
}

To cite: Zethof S, Bakker W, Nansongole F, et al. Pre-post implementation survey of a multicomponent intervention to improve informed consent for caesarean section in Southern Malawi. BMJ Open 2020;10:e030665. doi:10.1136/ bmjopen-2019-030665

\section{- Prepublication history and} additional material for this paper are available online. To view these files, please visit the journal online (http://dx.doi. org/10.1136/bmjopen-2019030665).

SZ and WB contributed equally.

Received 26 March 2019 Revised 29 August 2019 Accepted 18 0ctober 2019

A) Check for updates

(C) Author(s) (or their employer(s)) 2020. Re-use permitted under CC BY-NC. No commercial re-use. See rights and permissions. Published by BMJ.

${ }^{1}$ Department of Obstetrics and Gynaecology, Leiden University Medical Center, Leiden, The Netherlands

${ }^{2}$ Clinical Department, St. Luke's Hospital, Zomba, Malawi ${ }^{3}$ Athena Institute, Faculty of Science, VU University, Amsterdam, The Netherlands ${ }^{4}$ Nursing Department, St. Luke's Hospital, Zomba, Malawi

Correspondence to

Dr Wouter Bakker;

bakker.stlukes@gmail.com

\section{ABSTRACT}

Objective Surgical informed consent is essential prior to caesarean section, but potentially compromised by insufficient communication. We assessed the association between a multicomponent intervention and women's recollection of information pertaining to informed consent for caesarean section in a low-resource setting, thereby contributing to respectful maternity care.

Design Pre-post implementation survey, conducted from January to June 2018, surveying women prior to discharge.

Setting Rural 150-bed mission hospital in Southern Malawi.

Participants A total of 160 postoperative women were included: 80 preimplementation and 80 postimplementation.

Intervention Based on observed deficiencies and input from local stakeholders, a multicomponent intervention was developed, consisting of a standardised checklist, wall poster with a six-step guide and on-the-job communication training for health workers.

Primary and secondary outcome measures Individual components of informed consent were: indication, explanation of procedure, common complications, implications for future pregnancies and verbal enquiry of consent, which were compared preintervention and postintervention using $\chi^{2}$ test. Generalised linear models were used to analyse incompleteness scores and recollection of the informed consent process.

Results The proportion of women who recollected being informed about procedure-related risks increased from $25 / 80$ to $47 / 80$ (OR 3.13 (95\% Cl 1.64 to 6.00 )). Recollection of an explanation of the procedure changed from 44/80 to 55/80 (OR 1.80 (0.94 to 3.44)), implications for future pregnancy from $25 / 80$ to $47 / 80$ (1.69 (0.89 to $3.20)$ ) and of consent enquiry from $67 / 80$ to $73 / 80$ (OR 2.02 (0.73 to 5.37)). After controlling for other variables, incompleteness scores postintervention were $26 \%$ lower $(\operatorname{Exp}(\beta)=0.74 ; 95 \% \mathrm{Cl} 0.57$ to 0.96$)$. Recollection of common complications increased with 0.25 complications $(\beta=0.25 ; 95 \% \mathrm{Cl} 0.01$ to 0.49 ). Recollection of the correct indication did not differ significantly.

Conclusion Recollection of informed consent for caesarean section changed significantly in the postintervention group. Obtaining informed consent for caesarean section is one of the essential components of respectful maternity care.
Strengths and limitations of this study

Based on locally identified deficiencies in clinical practice.

- Use of generalised linear models to quantify effect of intervention.

- Convenient study design with limited resources: limited sample size and follow-up, no control group.

- Use of incompleteness rather than completeness score, to attain Poisson distribution.

\section{BACKGROUND}

Informed consent is key to medical practice and embedded in national and international standards such as the Code of Ethics and Professional Conduct of the Medical Council of Malawi, and the International Covenant on Civil and Political Rights. ${ }^{1-3}$ Valid informed consent is defined as being able to accept an intervention willingly after receiving adequate and comprehensible information about risks and benefits. ${ }^{4}$ It is a preoperative necessity for all surgical procedures including caesarean section (CS), the most frequently performed surgical intervention in many parts of the world. ${ }^{5}$ In obstetrics, explanation of procedures and seeking consent are associated with improved rating of birth experience, while non-consented care is seen as a deterrent to skilled birth care utilisation. ${ }^{36}$

Several reports have recognised weaknesses in the process of acquiring surgical informed consent for obstetric procedures, such as providing no explanation of the indication for surgery, procedure-related risks or the postoperative trajectory. ${ }^{7-16}$ Women may feel pressured into undergoing surgery when little information is provided or information is not understood. ${ }^{914}$ At the same time, they may experience the provision of informed consent as a bureaucratic procedure not primarily serving their own interests. ${ }^{8} \mathrm{~A}$ variety of 


\section{Pilot study, 3 weeks}

Evaluating self-designed questionnaire

Exploring discordance between answer options and women's responses Examine clarity of questions and answer options

\section{Preintervention phase, 10 weeks}

Exit surveys with questionnaire

Inclusion of 80 women post-CS

\section{Intervention development and implementation, 2 weeks}

Analysing data from preintervention phase

Development of multicomponent intervention according to gathered

data, input from local stakeholders and guidelines

Standardised checklist

Poster with six-step guidance

Communication training

\section{Postintervention phase, 8 weeks}

Exit surveys with questionnaire

Inclusion of 80 women post-CS

Figure 1 Flow chart of study design. CS, caesarean section.

factors influence information transfer and retention, as well as shared decision-making. Poor communication between woman and health worker may be compounded by language barriers, low education level on the side of the woman and by lack of consent-related knowledge or communication skills among health workers. ${ }^{17-19}$ Additionally, emergency situations in which the informed consent process takes place may not be conducive to information retention due to shortage of time, physical limitations, anxiety and pain. ${ }^{1320}$ To overcome such barriers, health workers must improve women's ability to participate in the decision-making process as fully as possible and as far as reasonably practicable. ${ }^{21} 22$ Information should be provided without use of medical terminology, adjusted to the language and understanding of the woman. It is preferentially given during pregnancy or, if during labour, between contractions. ${ }^{1721}$

Studies implementing interventions to improve informed consent for surgical procedures (including CS) in low-resource settings are scarce, with most literature focussing on elective procedures in high-income countries. ${ }^{823-25}$ However, there are examples of studies using multicomponent interventions focussing on non-abusive and respectful maternity care. ${ }^{26-30}$ The landscape analysis by Bowser and Hill identified non-consented care as one of the contributing factors to disrespect and abuse in childbirth, stating that 'there is a lack of routine patient information, communication and consent protocols for obstetric procedures' in regions all over the world. ${ }^{3}$ We postulated that a multicomponent intervention standardising the informed consent process could improve women's recollection of having consented to care and, in this way, their birth experience. Consenting to obstetric interventions including CS is one of the important elements in the broader concept of respectful maternity care.

The objective of our study was to assess recollection of informed consent prior to and after introducing a multicomponent intervention consisting of a checklist, a six-step informed consent guide and communication training for health workers involved in maternity care.

\section{METHODS}

\section{Study design, setting and participants}

This prospective pre-post implementation survey was performed between 1 January 2018 and 1 June 2018 in the maternity department of a rural mission hospital in the southern region of Malawi. Maternity staff comprised locally trained midwives, associate clinicians (nonphysician clinicians with a predominantly practical training of 4 years) and two Medical Doctors in Global Health and Tropical Medicine (MD GHTM), trained in the Netherlands. ${ }^{31}$ The maternity department provides services free-of-charge and has an average of 200 births per month. CS rate in the study period was $15.7 \%$ (82 out of 523 total births) in the preintervention phase, and $19.5 \%(81 / 415)$ in the postintervention phase. The hospital had one operating theatre available for all procedures. All women undergoing CS were eligible for inclusion. Elective CS was defined as CS planned prior to onset of labour, while in unplanned CS the decision was made during the first or second stage of labour. Exclusion criteria were inability to participate due to bad clinical condition, referral or death prior to survey, or unwillingness to participate. The informed consent process was initiated by the midwife on duty, a medical doctor or associate clinician. After CS had been performed, women were admitted for at least 72 hours in the postnatal ward for observation and discharged in case no complications arose. Figure 1 shows an overview of the study process. The study protocol is attached as a supplementary file (online supplementary file 1 ).

\section{Data collection}

Prior to implementation, 80 women were surveyed between 1 January and 15 March 2018 using a standardised questionnaire. Surveys were performed on the day of discharge by one of the authors (SZ), assisted by rotating nursing college students who had not been involved in direct care for the woman. Data related to timing of surgery, indication and whether it was an elective or unplanned procedure were extracted from the records. After this initial period, 2 weeks were allocated to intervention development and implementation. Subsequently, 80 additional women were included between 1 April and 1 June 2018.

\section{Intervention development and implementation}

Together with representatives of the maternity department, a multicomponent intervention was designed consisting of a standardised checklist, wall poster with 
Table 1 Definition of primary outcomes

\section{Completeness - which topics have been discussed preoperatively?}

\begin{tabular}{|c|c|}
\hline Indication & Indication for caesarean section. \\
\hline Procedure & $\begin{array}{l}\text { Transfer to theatre, lower abdominal incision, use of anaesthetics and possibly blood } \\
\text { products. }\end{array}$ \\
\hline Implications for future pregnancies & $\begin{array}{l}\text { Need to deliver in secondary health facility in subsequent pregnancies. } \\
\text { Advice to have bilateral tubal ligation after third caesarean section.* }\end{array}$ \\
\hline Consent & Written and verbal consent has been collected. \\
\hline Recollection of indication & Woman names indication for caesarean section as mentioned in her medical records. \\
\hline $\begin{array}{l}\text { Recollection of common } \\
\text { complications }\end{array}$ & $\begin{array}{l}\text { Score from } 0 \text { to } 3 \text {, woman picks the following common complications out of a list of six } \\
\text { options: } \\
\text { Extensive bleeding (>1000 mL); } \\
\text { Infection (wound infection, endometritis, peritonitis); } \\
\text { Extended recovery time as opposed to vaginal birth (3-day hospital admission and } \\
\text { no lifting for } 6 \text { weeks); } \\
\text { Other included options: leaving instruments in the abdomen, permanent paraplegia, } \\
\text { maternal death. } \dagger\end{array}$ \\
\hline
\end{tabular}

*Based on national consensus.

†Extracted from Litorp et al and pilot survey. ${ }^{11}$

a six-step informed consent guide and communication training of health workers. The interventions aimed at addressing deficiencies observed in the preintervention period and brought forward by local stakeholders. This involved inadequacy of risk discussion, both in approach and content, and lack of women's involvement in decision-making. Interventions were supposed to reinforce one another by repeating important information and implementing checklist and poster into the training. The intervention consisted of the following:

\section{A standardised checklist}

Lack of informed consent protocols resulted in this checklist for health workers encompassing five components of the informed consent process: indication for operation with benefits of the proposed procedure, elaboration on the procedure, discussion of associated risks, implications for future pregnancies and verbal consent enquiry (table 1). Components were based on the National Institute for Health and Care Excellence clinical guidelines on CS. ${ }^{32}$ This particular guideline was used for its international recognition and clear outline on women-centred care. One additional checkbox addressed the opportunity given to the woman to ask questions. The importance of providing a woman with such opportunity was stressed in the communication training. The checklist was integrated into the facility's preoperative form, thereby reassuring that the surgeon or midwife would bring the checklist along for consent enquiry. The original form only stated whether consent was given, without specifying what had been discussed during the consent process (online supplementary file 2).
Posters with a six-step informed consent guide

These posters were placed in every labour room at eye level and served as an additional reminder to maternity care providers to initiate the informed consent discussion. The guide accentuated risk discussion due to its inadequacy in the preintervention period. Frequently occurring risks were separated from rarer risks, following consent advice from the Royal College of Obstetricians and Gynaecologists. ${ }^{33}$ We emphasised that, although it was set up as a step-by-step guide, health workers apply information in accordance with women's needs and circumstances (online supplementary file 3).

\section{Communication training}

In the second week of development and implementation, a training session for clinical staff in the maternity department was organised. The training was established by the research team ( $\mathrm{SZ}, \mathrm{WB}, \mathrm{FN}, \mathrm{KK})$ and based on the Royal College of Obstetricians and Gynaecologists Clinical Governance Advice on obtaining valid informed consent, Medical Council of Malawi Code of Ethics and Professional Conduct and input from the clinical team. $^{21}$ The training consisted of an introduction into the theory of informed consent and a respectful womancentred approach during labour, followed by counselling methods, using the standardised checklist and poster. We highlighted timing of conversation, addressing uncertainties and questions and the importance of acquiring verbal consent. Role-play in settings of both elective and unplanned CS was performed and subsequent feedback given by other participants applying Pendleton's rules for professional feedback. ${ }^{34}$ The single training session 
was attended by 10 midwives, 6 associate clinicians and 2 MD GHTM. Not all rotating clinicians and midwives were present due to conflicting clinical duties. Questions from participants were addressed and participants invited to provide input into improving the consent guide.

Checklist and guide were discussed in a plenary session with all hospital staff, which provided an opportunity for additional adjustments. Health workers were then provided copies of checklist and guide. After the plenary session, posters were placed in the ward and use of the form with checklist started. No other interventions related to quality of care were implemented during the postintervention period.

\section{Study tool}

For the preimplementation and postimplementation surveys, an exit questionnaire was created in English and Chichewa using forward and subsequent backward translation (Online supplementary files 4,5). An expert committee consisting of experienced clinicians and midwives working in the maternity department of the hospital were involved in validating its content. This included how indications for CS should be grouped, which complications should be known to women and what information is indispensable with regard to future pregnancies. Additionally, participant and procedure related variables with potential impact on outcomes were identified. Use of medical terminology was reduced to ensure that all questions could easily be understood. A 3-week pilot study was performed, whereby in the first week women were asked open-ended questions to obtain insight in probable answers. Mentioned risks related to CS were noted and used as answer options in the later version of the questionnaire. In the following 2 weeks, clarity of the study tools was examined and the order of questions and answer options adjusted in order to be easily understood by participants.

\section{Outcome variables}

Primary study outcomes were level of incompleteness and recollection of common complications and indication (table 1). Incompleteness was defined as the number of informed consent components not discussed according to the woman. For each component, the woman was asked whether it was discussed during the consent process. Each of five components was dichotomously scored ( $1=$ not discussed, $0=$ discussed) and rated as equally important. This resulted in an 'incompleteness score' ranging from 5 (=none of the components discussed) to 0 (=all components discussed). An 'incompleteness' rather than a 'completeness' score was used, due to adoption of a Poisson distribution by the outcome variable. To assess recollection of common complications a list with complications was provided, of which three were commonly associated with CS and three were not. For every common complication mentioned, one point was given. Common complications deemed as essential knowledge for women in our setting were extensive bleeding of $>1 \mathrm{~L}$, infections such as wound infection, endometritis or peritonitis and an extended recovery time compared with vaginal birth. Three other choices were added to the list, based on complications named by women during the pilot study. Recollection of indication was measured by the percentage of women who described the indication for CS as stated in the medical records. Indications were categorised using plain, non-medical language such as 'problem with heart rate of the child' or 'high blood pressure'.

\section{Analytic approach}

For descriptive analyses, unpaired t-test, Mann-Whitney U test or $\chi^{2}$ test were used depending on the type of variable and normality of its distribution. For completeness, each individual component of informed consent was compared between the preintervention and postintervention groups using $\chi^{2}$ tests with ORs and $95 \%$ CIs. Additionally, generalised linear models were used to identify the attribution of the intervention on dependent variables: 'incompleteness score', 'number of recollected common complications' and 'correct indication recall percentages'. For the incompleteness scores, a Poisson regression was adopted, due to a Poisson distribution of the dependent variable (one sample independent KolmogorovSmirnov test $(\mathrm{p}=0.57))$. The model's goodness of fit (Pearson's $\chi^{2} / \mathrm{df}$ ) was 0.96 and the omnibus test showed a significant difference between the model and intercept model $(\mathrm{p}<0.001)$. Number of recollected complications was normally distributed according to Jarque-Bera test of $1.44(p>0.1)$ and a linear model was used. Goodness of fit was 0.61 and the omnibus test showed a significant difference $(p=0.03)$. Binomial logistic regression was used with correct indication recall percentages as dependent variable. Goodness of fit was 1.06 and omnibus test showed no statistically significant difference $(p=0.14)$. Type and timing of CS, antenatal consultations and prior CS were identified as explanatory variables based on the literature. ${ }^{1333} 35$ Additional explanatory independent variables were identified based on subsequent application of variables in the different models, and included when $\mathrm{p}<0.05$. Exponentiated regression coefficients $(\operatorname{Exp}(\beta))$ and their 95\% CIs were reported for the Poisson and logistic bivariate model, whereas for the linear model regression coefficients $(\beta)$ were reported. All analyses were performed with IBM SPSS V.24.0. Alpha was set at 0.05. Analysis and interpretation of data adhered to Standards for Quality Improvement Reporting Excellence 2.0 guidelines. ${ }^{36}$

\section{Ethical consideration}

Permission was granted by the hospital management to conduct the study. All participants were provided with an informed consent sheet either in English or Chichewa, regarding the purpose of the study, their right to stop participation at all times and a request to access their medical files. For women who were illiterate, the interview assistant read the consent form out loud and elaborated. Finger prints were accepted as signatures for women who did not know how to write. No names were 
included during data collection to ensure confidentiality. All women were asked to give informed consent before inclusion. Patient files were accessed only after approval was obtained. Patient records were brought with them to the exit survey and extracted data was linked to their anonymised study number. Immediately after collection, data were stored in a locally encrypted database, only accessible by the primary investigators.

\section{Patient and public involvement}

The importance of improving informed consent was highlighted in various hospital advisory committee meetings, in which local chiefs present concerns of the community. This laid the foundation for this study. During the pilot phase, women were asked to comment on study tools, in order to make these as easily understandable and applicable as possible.

\section{RESULTS}

During the study period, 163 women were eligible for inclusion, of whom 160 (98\%) participated. One woman was discharged before the scheduled interview and two refused to participate. All participating women completed the interview.

\section{Participant-related and procedure-related characteristics}

The majority of women had no previous CS; 54 (67.5\%) preintervention and postintervention (Table 2). In both groups, the highest percentage of women were aged between 20 and 24 years. Median age of the preintervention group was 26 (IQR 21-30) as compared with 24 (IQR $21-30 ; \mathrm{p}=0.96$ ) in the postintervention group. Inability to read English or Chichewa was observed in 17 (21.3\%) women preintervention and in $15(18.8 \%)$ postintervention. No statistically significant differences were found with regard to women's parity, antenatal consultations, highest educational level and religion. Daily occupation differed statistically significantly $(p<0.05)$, with more self-employed women in the preintervention group $(21,26.3 \%)$ compared with the postintervention group $(7,8.8 \%)$. The majority of CS were unplanned in both groups, $66(82.5 \%)$ and $68(85 \%)$. A statistically significant difference was observed in the attendance of medical doctors during CS: 12 CS (15\%) in the preintervention group compared with 37 CS $(46.3 \%)$ in the postintervention group. No statistically significant differences in timing of or indications for CS were found.

\section{Completeness of informed consent}

In the postintervention group, $47(58.8 \%)$ women expressed that they had received information on risks before surgery, as compared with $25(31.3 \%)$ in the preintervention group (OR 3.13; 95\% CI 1.64 to 6.00) (table 3). Changes in explanation of the procedure (OR $1.80 ; 95 \%$ CI 0.95 to 3.44 ), inclusion of implications for future pregnancies (OR 1.69; 95\% CI 0.89 to 3.20) and verbal consent enquiry (OR 2.02; $95 \%$ CI 0.73 to 5.37 ) were noted, although none of these was statistically significant. The component 'indication for the procedure' was mentioned equally in both groups (96.3\%). Independent variable analysis showed 'age' and 'ability to read English/Chichewa' to be significantly associated with incompleteness scores. No correlation was found with type of surgeon or daily occupation. Incompleteness scores were $26 \%$ lower in women surveyed after implementation of the intervention $(\operatorname{Exp}(\beta)=0.74 ; 95 \%$ CI 0.57 to 0.96 ) (table 4). Age was associated with a $4 \%$ decrease per year $(\operatorname{Exp}(\beta)=0.96 ; 95 \%$ CI 0.94 to 0.99$)$. Inability to read English or Chichewa provided 30\% higher incompleteness scores $(\operatorname{Exp}(\beta)=1.3 ; 95 \%$ CI 1.02 to 1.83$)$.

\section{Recollection of informed consent}

The linear model identified an increase of 0.25 recollected complications in the postintervention group when corrected for other variables ( $\beta=0.25 ; 95 \%$ CI 0.01 to 0.49 ) (table 5). Age of participants was identified as an additional explanatory variable and associated with 0.02 more common complications recalled per year ( $\beta=0.02 ; 95 \%$ CI 0.00 to 0.04$)$. Logistic binomial regression examined that women counselled postimplementation were 2.11 times more likely to recall the indication for $\operatorname{CS}(\operatorname{Exp}(\beta)=2.11$; $95 \%$ CI 0.96 to 4.60 ) (Table 6 ). No additional explanatory variables were identified to be associated with correct indication recall percentages.

\section{DISCUSSION}

This study evaluated a multicomponent intervention, consisting of an informed consent checklist, guide and training, providing standards and tools for the informed consent process prior to CS. The intervention had been developed and implemented in cooperation with clinical staff hoping to increase perceived acceptability, a necessary condition for effectiveness. ${ }^{37}$ Other community or system-related issues potentially influencing the intervention's effectiveness were normalisation of non-consented care, and lack of patient autonomy and legal redress mechanisms. ${ }^{3}$ Although these issues were touched on, the current intervention will not suffice as a complete solution. We opted for a prospective pre-post implementation study design because randomisation was not compatible with the study setting and preintervention data deemed to be necessary for development and implementation of the multicomponent intervention.

The percentage of women stated to have received information on procedure-related risks was $27.5 \%$ higher in the postintervention group. Furthermore, the procedure was explained more frequently and more women were able to reproduce the indication for CS, although this trend was not statistically significant. An explanation for the latter not reaching the level of statistical significance could be that the informed consent consultation in the preintervention group already included an explanation of the proposed procedure and implications for future pregnancies in considerably large, although still 
Open access

Table 2 Participant and procedure characteristics for the preintervention and postintervention group

\begin{tabular}{|c|c|c|c|}
\hline & $\begin{array}{l}\text { Preintervention } \\
(n=80)(\%)\end{array}$ & $\begin{array}{l}\text { Postintervention } \\
(n=80)(\%)\end{array}$ & $P$ value \\
\hline Age (years) & & & 0.96 \\
\hline $15-19$ & $16(20)$ & $14(17.5)$ & \\
\hline $20-24$ & $22(27.5)$ & $29(36.3)$ & \\
\hline $25-29$ & $21(26.3)$ & $12(15)$ & \\
\hline 30-34 & $15(18.7)$ & $14(17.5)$ & \\
\hline $35+$ & $6(7.5)$ & $11(13.8)$ & \\
\hline Median age $<\mid \mathrm{QR}>$ & $26<21-30>$ & $24<21-30>$ & \\
\hline Parity & & & 0.83 \\
\hline 1 & $31(38.8)$ & $31(38.8)$ & \\
\hline 2 & $21(26.3)$ & $18(22.5)$ & \\
\hline$>2$ & $28(34.9)$ & $31(38.8)$ & \\
\hline Prior CS & & & 0.24 \\
\hline 0 & $54(67.5)$ & $54(67.5)$ & \\
\hline 1 & $18(22.5)$ & $23(28.8)$ & \\
\hline$>1$ & $8(10)$ & $3(3.8)$ & \\
\hline Inability to read English/Chichewa (\%) & $17(21.3)$ & $15(18.8)$ & 0.69 \\
\hline Highest educational level attained & & & 0.06 \\
\hline No formal education & $7(8.8)$ & $6(7.5)$ & \\
\hline Primary school & $36(45)$ & $34(42.5)$ & \\
\hline Junior secondary school & $11(13.8)$ & $5(6.3)$ & \\
\hline Senior secondary school & $18(22.5)$ & $20(25)$ & \\
\hline College/University & $8(10)$ & $15(18.8)$ & \\
\hline Religion & & & 0.84 \\
\hline Christian & $40(50)$ & $41(51.3)$ & \\
\hline Jehovah's witness & $2(2.5)$ & $1(1.3)$ & \\
\hline Muslim & $38(47.5)$ & $38(47.5)$ & \\
\hline Occupation & & & $<0.05$ \\
\hline Employed & $8(10)$ & $12(15)$ & \\
\hline Business/self-employed & $21(26.3)$ & $7(8.8)$ & \\
\hline Student & $3(3.8)$ & $3(3.8)$ & \\
\hline Housewife & 29 (36.3) & $27(33.8)$ & \\
\hline Farmer & $19(23.8)$ & $31(38.8)$ & \\
\hline Mean number of antenatal consultations \pm SD & $3.7+/-1.1$ & $3.53 \pm 1.1$ & 0.25 \\
\hline Timing of CS & & & 0.42 \\
\hline 08:00-18:00 hours & $46(57.5)$ & $51(63.7)$ & \\
\hline 18:00-08:00 hours & $34(42.5)$ & $29(36.3)$ & \\
\hline Type of CS & & & 0.67 \\
\hline Elective CS & $14(17.5)$ & $12(15)$ & \\
\hline Unplanned CS & $66(82.5)$ & $68(85)$ & \\
\hline Prevalence of indication categories & & & 0.19 \\
\hline Obstructed labour & $45(56.3)$ & $49(61.3)$ & \\
\hline Non-reassuring fetal status & $7(8.8)$ & $3(3.8)$ & \\
\hline Malposition/malpresentation & $6(7.5)$ & $12(15)$ & \\
\hline Pre-eclampsia/HELLP & $2(2.5)$ & $1(1.3)$ & \\
\hline
\end{tabular}




\begin{tabular}{|c|c|c|c|}
\hline & $\begin{array}{l}\text { Preintervention } \\
(\mathrm{n}=80)(\%)\end{array}$ & $\begin{array}{l}\text { Postintervention } \\
(\mathrm{n}=80)(\%)\end{array}$ & $P$ value \\
\hline Antepartum haemorrhage & $3(3.8)$ & $0(0)$ & \\
\hline Uterine rupture & $2(2.5)$ & $1(1.3)$ & \\
\hline$\geq 2$ CS in history & $8(10)$ & $3(3.8)$ & \\
\hline \multicolumn{4}{|l|}{ Surgeon performing CS } \\
\hline MD GHTM & $12(15)$ & $37(46.3)$ & $<0.05$ \\
\hline Clinical officer & $68(85)$ & $43(53.7)$ & \\
\hline
\end{tabular}

*Including (preterm) prelabour rupture of membranes, on woman's request.

CS, caesarean section; HELLP, Hemolysis, Elevated Liver Enzymes, Low Platelet count, a complication associated with (pre-)eclampsia; MD GHTM, Medical Doctors in Global Health and Tropical Medicine.

deficient, proportions. Additional and more specific measures may be required to further improve recollection of these items. The supplementary poster mainly focused on risk-discussion, possibly overlooking other components. Consent enquiry was incomplete in both groups, which in every case was explained by absence of verbal consent. This is a major concern, since surgery should not be performed without consent. After controlling for other explanatory independent variables, incompleteness scores were $26 \%$ lower in women counselled postintervention. This implies that more components of informed consent were included after implementation. The variables 'attended by MD GHTM' and 'daily occupation' differed preintervention and postintervention, but no association with incompleteness scores was found in the multivariate model. A higher age of the woman, however, was associated with lower incompleteness scores, even after correcting for parity and the presence of prior CS. Possibly younger women experience discriminatory behaviour based on providers' prejudice, as has been reported previously. ${ }^{3}$ Additionally, young women might be less involved in decision making when seniors are present to speak for them. ${ }^{19}{ }^{38}$ Age and inability to read Chichewa or English resulted in higher incompleteness scores. This underlines the need for verbal explanation and consent enquiry in addition to the written consent form. Written consent forms should be made available in local languages.

Besides more risk discussions being included during the informed consent process, an increase of the number of recalled risks was observed postintervention, suggesting an increased recollection of common complications. Despite its statistical significance, the effect size was considered to be small. Higher correct indication recall percentages were seen, although this did not reach the level of statistical significance. It is important that information is reproducible. A signed consent form may not be valid if information has not been understood. ${ }^{39} 40$ Women's educational level, language competency and provider's effective communication of procedure, risks and recovery have previously been identified as important determinants to comprehend the informed consent process. ${ }^{40}{ }^{41}$ Despite inclusion of more informed consent components postimplementation, discrepancies may exist between provider and women's perspectives of the informed consent process. ${ }^{42}$ Written material in women's vernacular may increase understanding, but written consent forms were previously found to be difficult to understand by women going for unplanned obstetric surgery. ${ }^{913}$

Table 3 Completeness of informed consent; number of informed consent aspects discussed during preoperative counselling

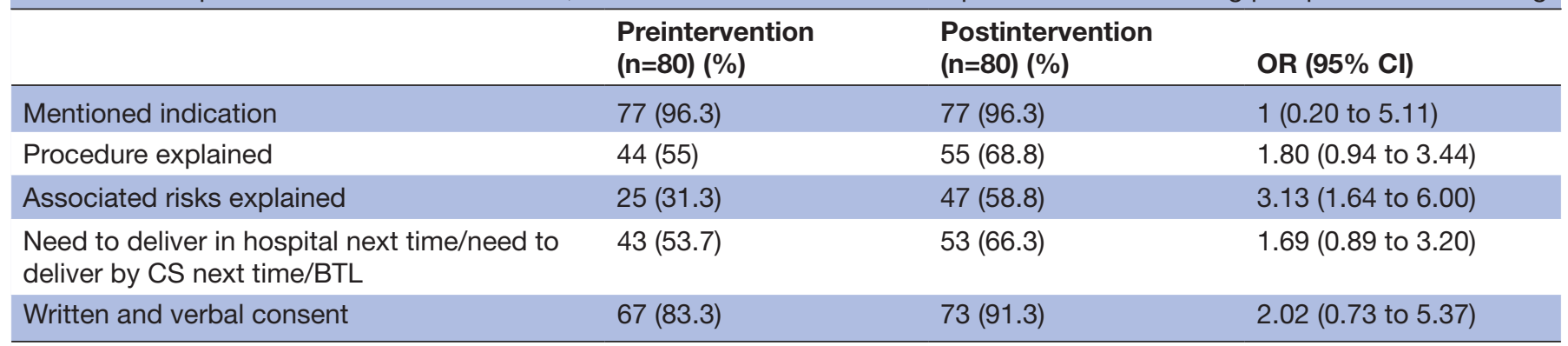

Comparison between preintervention and postintervention group.

BTL, bilateral tubal ligation (female sterilization); CS, caesarean section. 
Table 4 Generalised linear model: Poisson

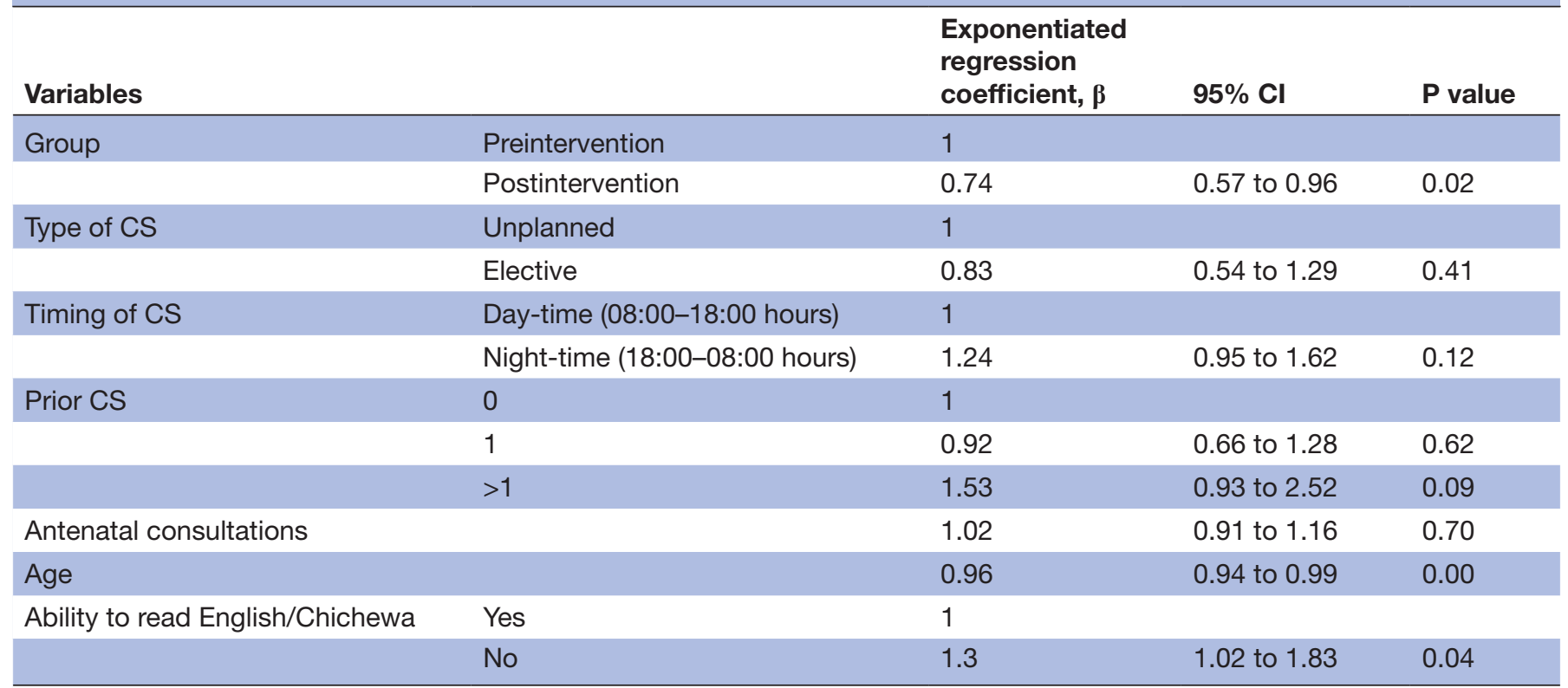

Variables associated with incompleteness scores.

CS, caesarean section.

Efforts were made to sustain motivation and participation by including verbal consent as one of the five components and giving women and their guardians an opportunity to ask for clarification. Involvement in the informed consent process may give women the feeling of being in control and enhance their relationship with healthcare providers. These are two facilitators of a positive birth experience. ${ }^{43}$ In addition to standardisation, we measured outcomes at patient level, which is an indirect reflection of interventions at health system level. Interference of woman-related factors such as prior experiences, emotional barriers and physical impairment may occur, and may not be covered by our intervention. Nevertheless, the quality of informed consent is reflected in women's recollection.

Our chosen study design has several limitations. First, given the uncontrolled pre-post study design conclusions with regard to causality between intervention and studied outcome are impossible. Study groups were different with regard to daily occupation and type of surgeon. Although these particular variables were not independently statistically significantly associated with the outcome, the latter could have been confounded by co-occurring contextual differences, such as policy changes and acquaintance with the research team. ${ }^{44}$ Several potential confounders were included in the model and the research team was stable

\begin{tabular}{|c|c|c|c|c|}
\hline Variables & & Regression coefficient, $\beta$ & $95 \% \mathrm{Cl}$ & $P$ value \\
\hline Group & Preintervention & 0 & & \\
\hline \multirow[t]{2}{*}{ Type of CS } & Unplanned & 0 & & \\
\hline & Elective & 0.27 & -0.09 to 0.63 & 0.14 \\
\hline & Night-time (18:00-08:00 hours) & -0.02 & -0.28 to 0.23 & 0.86 \\
\hline \multirow[t]{3}{*}{ Prior CS } & 0 & 0 & & \\
\hline & 1 & 0.06 & 0.23 to 0.34 & 0.69 \\
\hline & $>1$ & -0.18 & 0.68 to 0.33 & 0.49 \\
\hline Antenatal consultations & & 0.10 & -0.14 to 0.21 & 0.09 \\
\hline
\end{tabular}

Variables associated with number of recollected common complications.

CS, caesarean section. 
Table 6 Generalised linear model: binary logistic

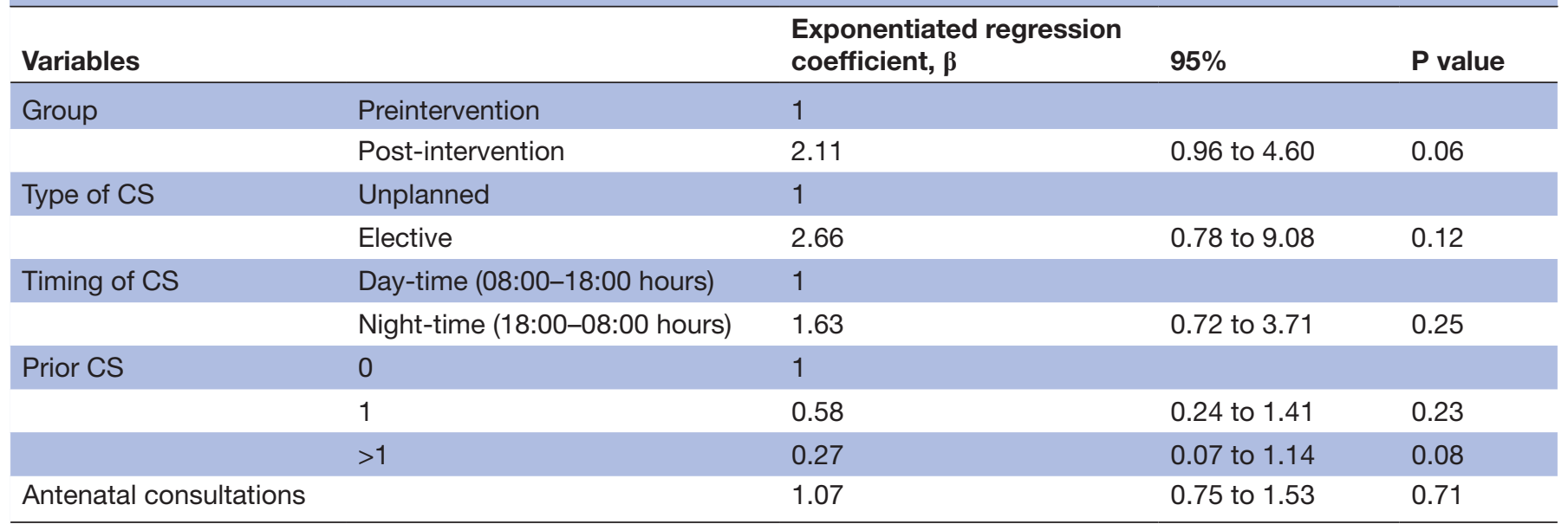

Variables associated with correct indication recall percentages.

CS, caesarean section.

throughout the study period, therefore we think that residual confounding and researcher bias are limited, but these cannot be excluded. Time elapse between preimplementation and postimplementation phases was minimised, no additional interventions were implemented at facility level and no interventions were reported by local government at the time. While the limited time elapse between both groups may be beneficial to reduce the chance that concurring events influence outcomes, it may complicate assessing sustainability of the intervention, since the effect is measured shortly after implementation. Second, a sample size calculation was not performed due to absence of prevalence data on informed consent recollection in our setting or similar populations in the designing phase of the study. At the time of finalising this paper, such data are available. ${ }^{78}$ Our sampling was based on convenience and logistical possibilities. Third, due to the use of a Poisson regression analysis, 'incompleteness' rather than 'completeness' scores were used, increasing goodness of fit of the model, but rendering interpretation possibly more difficult. Additional limitations were incomplete validation of our self-designed questionnaire with regard to test-retest reliability, inter-rater reliability and the tool's responsiveness to changes in outcome, and existing language barriers between interviewer and participants. To diminish these effects, we designed the questionnaire to be simple and give little room for interpretation, with both multiple choice and closed-ended questions. When necessary, translation was done by local nursing college students. The presence of health workers might have led to socially desirable answers, although none of the participating students were involved in the consent process or birth.

In future research, attribution of the intervention to the observed difference in recollection of informed consent has to be confirmed by including a control group in the study design. Outcomes other than completeness of the consultation and women's recollection are worth investigating. New studies could explore influence of our multicomponent intervention on women's satisfaction, anxiety and long-term comprehension, and this intervention or similar context-specific interventions should be assessed in other settings.

\section{CONCLUSION}

After implementation of a multicomponent intervention recollection of the informed consent process for CS improved. Women stated more frequently to have received information on the procedure, possible complications and implications for future pregnancies. Recollection of common complications was significantly higher postintervention. These results suggest that standardisation and training positively influence informed consent in a resource-poor setting, and thereby promote respectful maternity care.

Acknowledgements The authors would like to thank all participating staff. In particular, the authors would like to thank the nursing students who were involved during interviews, A. Musukwa for assisting with translation of the questionnaire and A.C. Huigens for providing managerial assistance.

Contributors SZ and WB drafted the study protocol, with help of TvdA and JvR. FN and KK provided feedback on the study design and helped implementing the study in the facility. SZ and FN drafted the study tool. KK assisted in inclusion and logistics around the interviews. SZ conducted the interviews analysed the data together with WB. SZ and WB drafted the manuscript which was commented on by all authors and approved for submission.

Funding The authors have not declared a specific grant for this research from any funding agency in the public, commercial or not-for-profit sectors.

Competing interests None declared.

Patient consent for publication Not required.

Ethics approval The study received ethical approval by the National Health Sciences Research Committee of Malawi (approval number 1995) and a declaration of no-objection by the Leiden University Medical Centre Ethical Committee (reference number P18.027).

Provenance and peer review Not commissioned; externally peer reviewed.

Data availability statement Anonymized and non-traceable data are available in a public, open-access repository. 
Open access This is an open access article distributed in accordance with the Creative Commons Attribution Non Commercial (CC BY-NC 4.0) license, which permits others to distribute, remix, adapt, build upon this work non-commercially, and license their derivative works on different terms, provided the original work is properly cited, appropriate credit is given, any changes made indicated, and the use is non-commercial. See: http://creativecommons.org/licenses/by-nc/4.0/.

\section{REFERENCES}

1 Jolivet R. Respectful maternity care: the universal rights of childbearing women (full charter). White Ribbon Alliance, 2011. Available: http://www.healthpolicyproject.com/pubs/46_FinalRespect fulCareCharter.pdf [Accessed November 2018].

2 Medical Council of Malawi. Section 5.6: managing patients without informed consent. code of ethics and professional conduct 1990. Available: http://www.medicalcouncilmw.org/mcm/ethics.php [Accessed November 2018].

3 Bowser D, Hill K. Exploring evidence for Disrespect and abuse in Facility-Based childbirth: report of a landscape analysis. Washington, DC: Harvard School of Public Health and University Research Co, 2010.

4 Appelbaum PS. Assessment of patients' competence to consent to treatment. N Engl J Med 2007;357:1834-40.

5 Weiser TG, Haynes AB, Molina G, et al. Size and distribution of the global volume of surgery in 2012. Bull World Health Organ 2016:94:201-9.

6 Kambala C, Lohmann J, Mazalale J, et al. How do Malawian women rate the quality of maternal and newborn care? experiences and perceptions of women in the central and southern regions. BMC Pregnancy Childbirth 2015;15:169.

7 Teshome M, Wolde Z, Gedefaw A, et al. Surgical informed consent in obstetric and gynecologic surgeries: experience from a comprehensive teaching hospital in southern Ethiopia. BMC Med Ethics 2018;19:38.

8 Teshome M, Wolde Z, Gedefaw A, et al. Improving surgical informed consent in obstetric and gynaecologic surgeries in a teaching hospital in Ethiopia: a before and after study. BMJ Open 2019;9:e023408.

9 Habiba M, Jackson C, Akkad A, et al. Women's accounts of consenting to surgery: is consent a quality problem? Qual Saf Health Care 2004;13:422-7.

10 Biraboneye S P, Ogutu O, van Roosmalen J, et al. Trial of labour or elective repeat caesarean delivery:are women making an informed decision at Kenyatta national hospital? BMC Pregnancy Childbirth 2017; $17: 260$

11 Litorp H, Mgaya A, Kidanto HL, et al. 'What about the mother?' Women's and caregivers' perspectives on caesarean birth in a low-resource setting with rising caesarean section rates. Midwifery 2015;31:713-20

12 Ogunbode O, Oketona O, Bello F. Informed consent for caesarean section at a Nigerian university teaching hospital: patients perspective. Trop J Obstet Gynaecol 2015;32:55-63.

13 Akkad A, Jackson C, Kenyon S, et al. Informed consent for elective and emergency surgery: questionnaire study. BJOG 2004;111:1133-8.

14 Odhiambo A. "Stop making excuses": accountability for maternal health care in South Africa. New York: Human Rights Watch, 2011. Available: https://www.hrw.org/report/2011/08/08/stop-makingexcuses/accountability-maternal-health-care-south-africa [Accessed November 2018]

15 Kirane AG, Gaikwad NB, Bhingare PE, et al. "Informed" Consent: An Audit of Informed Consent of Cesarean Section Evaluating Patient Education and Awareness. J Obstet Gynecol India 2015;65:382-5.

16 Rajgire J, Vijay N. Consent for cesarean section: how much is informed? Panacea Journal of Medical Sciences 2017;7:62-4.

17 ACOG Committee opinion no. 585: health literacy. Obstet Gynecol 2014;123:380-3.

18 Osime OC, Okojie O, Osadolor F, et al. Current practices and medico-legal aspects of pre-operative consent. East Afr Med J 2004;81:331-5

19 Ezeome ER, Marshall PA. Informed consent practices in Nigeria. Dev World Bioeth 2009;9:138-48.
20 Hoehner PJ. Ethical aspects of informed consent in obstetric anesthesia-new challenges and solutions. J Clin Anesth 2003;15:587-600.

21 Royal College of Obstetricians \& Gynaecologists. Clinical governance advice No. 6: obtaining valid consent, 2015.

22 Falconer C. Mental capacity act 2005 code of practice. TSO London. Available: https://assets.publishing.service.gov.uk/government/ uploads/system/uploads/attachment_data/file/497253/Mentalcapacity-act-code-of-practice.pdf [Accessed May 2019].

23 Kinnersley P, Phillips K, Savage K, et al. Interventions to promote informed consent for patients undergoing surgical and other invasive healthcare procedures. Cochrane Database Syst Rev 2013;40.

24 Firdouse M, Wajchendler A, Koyle M, et al. Checklist to improve informed consent process in pediatric surgery: a pilot study. J Pediatr Surg 2017;52:859-63.

25 Kondziolka DS, Pirris SM, Lunsford LD. Improving the informed consent process for surgery. Neurosurgery 2006;58:1184-9.

26 Sheferaw ED, Bazant E, Gibson H, et al. Respectful maternity care in Ethiopian public health facilities. Reprod Health 2017;14:60.

27 Kujawski SA, Freedman LP, Ramsey K, et al. Community and health system intervention to reduce disrespect and abuse during childbirth in Tanga region, Tanzania: a comparative before-and-after study. PLoS Med 2017;14:e1002341.

28 Abuya T, Ndwiga C, Ritter J, et al. The effect of a multi-component intervention on disrespect and abuse during childbirth in Kenya. BMC Pregnancy Childbirth 2015;15:224.

29 Ratcliffe HL, Sando D, Lyatuu GW, et al. Mitigating disrespect and abuse during childbirth in Tanzania: an exploratory study of the effects of two facility-based interventions in a large public hospital. Reprod Health 2016;13:79.

30 Warren C, Njuki R, Abuya T, et al. Study protocol for promoting respectful maternity care initiative to assess, measure and design interventions to reduce disrespect and abuse during childbirth in Kenya. BMC Pregnancy Childbirth 2013;13:21.

31 Jiskoot P. On-the-job training of clinical officers in Malawi. Mal Med $J$ 2008;20:74-7.

32 National Institute for Health and Care Excellence. Clinical guideline CG13: caesarean section, 2011. Available: https://www.nice.org.uk/ guidance/cg132 [Accessed November 2017].

33 Royal College of Obstetricians and Gynaecologists. Consent advice No. 7: caesarean section, 2009. Available: https://www.rcog.org. uk/en/guidelines-research-services/guidelines/consent-advice-7 [Accessed November 2018]

34 Chowdhury RR, Kalu G. Learning to give feedback in medical education. Obstet Gynaecol 2004;6:243-7.

35 Nurok M, Czeisler CA, Lehmann LS, et al. Sleep deprivation, elective surgical procedures, and informed consent. N Engl J Med 2010;363:2577-9.

36 Ogrinc G, Davies L, Goodman D, et al. Squire 2.0 (standards for quality improvement reporting excellence): revised publication guidelines from a detailed consensus process. BMJ Qual Saf 2016;25:986-92

37 Sekhon M, Cartwright M, Francis JJ. Acceptability of healthcare interventions: an overview of reviews and development of a theoretical framework. BMC Health Serv Res 2017;17:88.

38 Hoffman M, Mofolo I, Salima C, et al. Utilization of family members to provide hospital care in Malawi: the role of hospital guardians. Malawi Med J 2012;24:74-8.

39 Department of Health and Social Care. Reference guide to consent for examination or treatment, second edition. 2009. Available: https:// webarchive.nationalarchives.gov.uk/20130123231708/http://www.dh. gov.uk/en/DH_103643 [Accessed May 2019]

40 Sherlock A, Brownie S. Patients' recollection and understanding of informed consent: a literature review. ANZ J Surg 2014;84:207-10.

41 Chan T, Eckert K, Venesoen P, et al. Consenting to blood: what do patients remember? Transfus Med 2005;15:461-6.

42 Friedman M, Arja W, Batra R, et al. Informed consent for blood transfusion: what do medicine residents tell? what do patients understand? Am J Clin Pathol 2012;138:559-65.

43 Hodnett ED. Pain and women's satisfaction with the experience of childbirth: a systematic review. Am J Obstet Gynecol 2002;186:S160-72.

44 Ho AMH, Phelan R, Mizubuti GB, et al. Bias in before-after studies: narrative overview for Anesthesiologists. Anesth Analg 2018;126:1755-62. 